OPEN ACCESS

Edited by:

Wei Yang,

Peking University Cancer

Hospital, China

Reviewed by:

Hong Wang,

Peking University Cancer

Hospital, China

Jie Wu,

Guizhou Provincial People's

Hospital, China

${ }^{*}$ Correspondence:

Yasunori Minami

minkun@med.kindai.ac.jp

Specialty section: This article was submitted to

Cancer Imaging and Image-directed Interventions,

a section of the journal

Frontiers in Oncology

Received: 11 August 2020

Accepted: 19 January 2021

Published: 05 March 2021

Citation:

Minami Y and Kudo M (2021) Image

Guidance in Ablation for Hepatocellular

Carcinoma: Contrast-Enhanced Ultrasound and Fusion Imaging.

Front. Oncol. 11:593636.

doi: 10.3389/fonc.2021.593636

\section{Image Guidance in Ablation for Hepatocellular Carcinoma: Contrast-Enhanced Ultrasound and Fusion Imaging}

\author{
Yasunori Minami ${ }^{*}$ and Masatoshi Kudo \\ Department of Gastroenterology and Hepatology, Faculty of Medicine, Kindai University, Osaka, Japan
}

The ultrasound (US) imaging technology, including contrast-enhanced US (CEUS) and fusion imaging, has experienced radical improvement, and advancement in technology thus overcoming the problem of poor conspicuous hepatocellular carcinoma (HCC). On CEUS, the presence or absence of enhancement distinguishes the viable portion from the ablative necrotic portion. Using volume data of computed tomography (CT) or magnetic resonance imaging (MRI), fusion imaging enhances the three-dimensional relationship between the liver vasculature and HCC. Therefore, CT/MR-US fusion imaging provides synchronous images of CT/MRI with real-time US, and US-US fusion imaging provides synchronous US images before and after ablation. Moreover, US-US overlay fusion can visualize the ablative margin because it focuses the tumor image onto the ablation zone. Consequently, CEUS and fusion imaging are helpful to identify HCC with little conspicuity, and with more confidence, we can perform ablation therapy. CEUS/fusion imaging guidance has improved the clinical effectiveness of ablation therapy in patients with poor conspicuous HCCs. Therefore; this manuscript reviews the status of CEUS/fusion imaging guidance in ablation therapy of poor conspicuous HCC.

Keywords: ablation therapy, contrast-enhanced ultrasound, fusion imaging, hepatocellular carcinoma, poor conspicuity, precise ablation

\section{INTRODUCTION}

Ablation therapy is a minimally invasive treatment option, and percutaneous ultrasound (US)guided ablative treatments, including radiofrequency ablation (RFA), and microwave ablation (MWA), have successfully managed hepatocellular carcinoma (HCC) (1-6). However, patients with difficult conditions for ablation therapy require multiple treatment sessions due to the limitation of US guidance. Poorly conspicuous HCC is not easily targeted on B-mode US guidance and accounts for $5.2-38.8 \%$ of planning US for ablation therapy $(7-10)$. The success of

Abbreviations: CEUS, contrast-enhanced ultrasound; CT, computed tomography; HCC, hepatocellular carcinoma; MPR, multiplanar reconstruction; MRI, magnetic resonance imaging; MWA, microwave ablation; RFA, radiofrequency ablation; US, ultrasound; 2D, two-dimensional; 3D, three-dimensional. 
percutaneous ablation therapies primarily depends on correct targeting through an imaging technique and the suitable placement of the needle electrode into the target tumor thereby optimizing local tumor control.

The US imaging technology has experienced radical improvement, and advances in hardware and software have helped to overcome the problem of poor conspicuity on US. Presently, contrast-enhanced ultrasound (CEUS) is widely used in clinical practice and it provides significant contribution to the diagnosis of HCC $(11,12)$. On CEUS, we can distinguish the viable portion of HCC from the ablative necrotic one by the presence or absence of enhancement, and we can perform an image-guided ablation of this viable HCC. In addition, other technological advancements allow two-dimensional (2D) multiplanar reconstruction (MPR) images of CT or MRI to display in the same plane as US images. Consequently, fusion imaging becomes a powerful technique to detect poor conspicuous HCC on US. Moreover, image fusion technology contributes to the progress of ablation therapy as well as other fields.

This article reviews the principles, clinical applications, and techniques of US image-guidance in ablation therapy including CEUS and fusion imaging.

\section{CEUS-GUIDED ABLATION}

\section{Contrast Agents and Pharmacokinetics}

Contrast agents, such as SonoVue/Lumason (Bracco, Milan, Italy) and Sonazoid (GE Healthcare, Waukesha, WI, USA), are microbubbles containing a low-solubility gas enveloped by a phospholipid shell. These microbubbles provide stable nonlinear oscillation in a low-power acoustic field because of their hard shells, thereby displaying vascular pattern in real-time. However, Kupffer cells of the liver engulf the contrast agent (especially sonazoid). Therefore, sonazoid microbubbles can accumulate in the liver parenchyma, thereby displaying enhancement of the liver parenchyma for a considerable period (13).

Regarding CEUS, the standard protocol for the examination of the liver consists of two main phases: the vascular and Kupffer phases (14-17). The vascular phase can be divided into three phases. These include the arterial phase (15 s after injection and lasting for 25-30 s), portal phase (30 s after injection and lasting for 2-3 $\mathrm{min}$ ), and late vascular phase (4-7 min). The Kupffer phase starts 10 min post-injection of Sonazoid. The key diagnostic feature of HCC with SonoVue/ Lumason is the hyper-enhancement seen in the arterial phase followed by a clearance seen in the portal and/or late phase. Similarly, we observe a hyper-enhancement in the arterial phase followed by defect in the Kupffer phase with sonazoid. In addition, repeated contrast injections are also useful for diagnosis of HCC. This procedure termed "defect reperfusion imaging" or "the re-injection technique" can diagnose HCC in the presence of arterial enhancement(s) in a defective lesion/ wash-out (18-20).

\section{Technique of CEUS Guidance}

Prior to ablation therapy, we can use CEUS to assess the HCC lesion size, number, margins, and relationship with the surrounding liver vasculature. The diagnostic accuracy of CEUS (using SonoVue or Sonazoid) for poorly conspicuous HCC is $93.8-100 \%$, similar to contrast-enhanced CT or MRI (21-24). Thus, CEUS facilitates needle placement in HCC poorly conspicuous on B-mode US, such that the defect/wash-out lesion signifies the target insertion point. Moreover, we can administer US contrast agents repeatedly in order to guide percutaneous ablation of multiple lesions.

Sometimes, it is difficult to differentiate local HCC tumor progression from ablative necrotic areas because both similarly show hypoenhancement lesions in the late vascular/Kupffer phase. Consequently, the defect reperfusion imaging becomes very useful in the confirmation of viable HCCs that are otherwise undetectable on US (20). Nevertheless, it is sometimes difficult to depict HCC with low-contrast images in the late vascular/ Kupffer phase in severe liver cirrhosis. In other words, we experience a weak contrast brightness intensity of liver parenchyma due to a decrease of portal blood flow and number of Kupffer cells in the liver. Therefore, in such situations, we must administer a higher dose of US contrast agent to patients in order to improve contrast brightness intensity.

\section{Evidence of CEUS-Guided Ablation Therapy}

CEUS guidance in ablation therapy has increased operators' confidence and improved the outcome. It was reported that the success rate at first session of CEUS was significantly higher than that in B-mode US guidance for poor conspicuous HCC (94.7 versus $65.0 \%, \mathrm{p}=0.043$ ) (25). Some previous cohort studies found that the number of sessions was significantly smaller with CEUS guidance than with B-mode US guidance $(26,27)$. Another study demonstrated that the local control rate was higher with CEUS guidance than with B-mode US guidance (85.3 versus $66.4 \%$ at 2 years, $\mathrm{p}=0.044)(28)$. Moreover, CEUS easily recognizes serious acute complications including active bleeding or hepatic infarction, which is not the case with B-mode US guidance (29). CEUS may show active hemorrhage as extravasation of microbubbles through the needle tract and hepatic infarction as a hypoenhancement lesion.

\section{FUSION IMAGING-GUIDED ABLATION}

\section{Applications of Fusion Imaging}

The commercial image fusion platforms include Real-Time Virtual Sonography (RVS) (Hitachi, Tokyo, Japan), volume navigation (v-nav) (GE Healthcare, Waukesha, WI, USA), SmartFusion (Canon Medical systems, Tokyo, Japan), eSie Fusion Imaging (Siemens Healthcare, Erlangen, Germany), and PercuNav (Philips, Andover, MA, USA).

Cross-sectional MPR images from 3D-volume data allow virtual sonographic images, and magnetic tracking based on mapping of a 3D magnetic field. When using fusion imaging, 


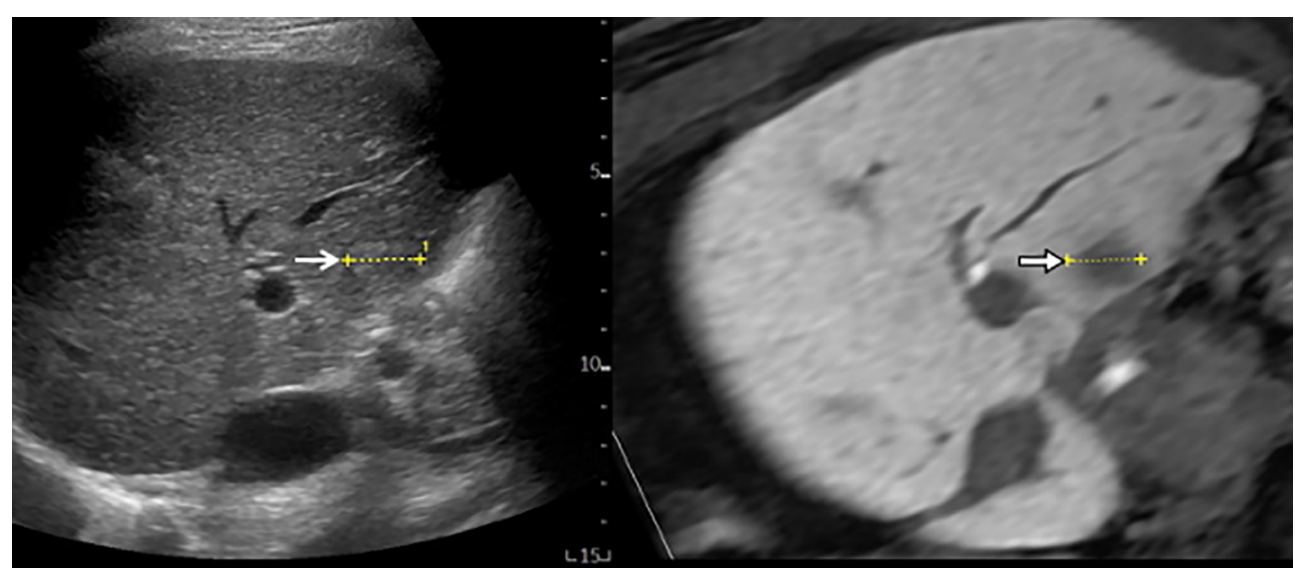

FIGURE 1 | MR-US fusion imaging. MRI and US images with HCC measuring $1.8 \mathrm{~cm}$ in diameter are well-matched. B-mode US (left) shows a slightly hyperechoic nodule with ill-defined HCC (open arrow) from intercostal view. Hepatobiliary phase image of gadoxetic acid-enhanced MRI (right) shows low signal intensity with illdefined HCC (arrow).

we can obtain spatial information from the relationship between the magnetic field generator and the magnetic sensor attached to the US probe. By integrating the spatial information between the US probe and $3 \mathrm{D}$ volume data, a $2 \mathrm{D}$-MPR image can show the same plane and move synchronously with real-time US images (30-32). CT/MR-US fusion imaging provides synchronous images of CT/MRI with real-time US (Figure 1). US-US fusion imaging provides synchronous US images before and after ablation (Figure 2). Moreover, it can visualize the ablative margins on US before ablation because it projects the tumor image onto the ablation zone.

\section{Technique of Fusion Imaging Guidance}

To operate effectively using fusion imaging technology, there is a need to match (co-register) the 3D image datasets with the real-time US; that is, we need to register the reference points near the tumor carefully. When the CT/MRI and US images are well-matched, inconspicuous HCC can be identified. Thus, CT/MR-US fusion imaging can increase the detectability of small HCCs compared to B-mode US. However, the gap in co-registration can persist in some situations. For example, imaging gap occurs because the depths of breath-holds in CT and sonographic examinations vary. Originally, CT/MR-US fusion imaging offer no support for synchronized action of breathing with any diagnostic US scanners at present. However, the priority is for the operator to catch the tumor location on US because the operator inserts the therapeutic needle watching on an US monitor under patients' breathing. Not so strictly image matching is necessary for inserting the therapeutic needle into the tumor in fact.

In contrast, succinct co-registration accuracy (in $\mathrm{mm}$ ) can be required when assessing an ablative margin. For high quality images, a 3D-UD volume has to be obtained by a swing scanning with slow and steady speed. For high-quality image matching, we need to register the reference points near the tumor before and after the ablation more carefully.

\section{Evidence of Fusion Imaging-Guided Ablation Therapy CT/MR-US Fusion Imaging}

According to some retrospective studies, the success and local tumor progression rates using RFA guided by CT/MR-US fusion imaging (for poorly conspicuous HCC on B-mode US) were 94.4$100 \%$ and $0-8.3 \%$, respectively (33-37). According to a prospective study by Ahn et al. (38), CT/MR-US fusion imaging significantly improved the tumor visibility and operators' confidence compared to B-mode US alone $(\mathrm{p}<0.001)$. Consequently, the recurrence-free survival rates were 86.0 and $75.8 \%$ at 12 - and 24 -months, respectively. The cumulative incidences of local tumor progression were 3.2 and $4.7 \%$ at 12 - and 24 -months, respectively.

\section{US-US Fusion Imaging and US-US Overlay Fusion}

Successful ablation therapy requires a wide ablation zone (including the tumor with ablative safety margin) in order to restrain local tumor progression. Although fusion imaging improves the visualization of HCC, some factors limit a 5-mm safety margin. These include large tumor size, tumor morphology, vasculature around the tumor, subcapsular tumor location, and gas bubbles in ablation zone $(39,40)$. Gas formation in particular, could envelope the tumor leading to a blind assessment of the ablative margin on US. Therefore, this ablative margin assessment technique was revised in order to overcome this challenging issue of a $5-\mathrm{mm}$ safety margin.

US-US fusion imaging is used to compare images before and after ablation in a side-by-side manner, and US-US overlay fusion visualizes the ablative margin by focusing on the ablation zone of the projected tumor image (41-45). We achieved 5-mm safety margins in $89.3 \%(108 / 121)$ of HCC nodules using the US-US overlay fusion technique compared to $47.0 \%(213 / 453)$ in the conventional guidance group $(\mathrm{P}<0.01)$. Two-year local tumor progression rates were $0.8 \%(1 / 121)$ with US-US overlay fusion and $6.0 \%(27 / 453)$ with conventional guidance $(\mathrm{P}=0.022)(46)$. 


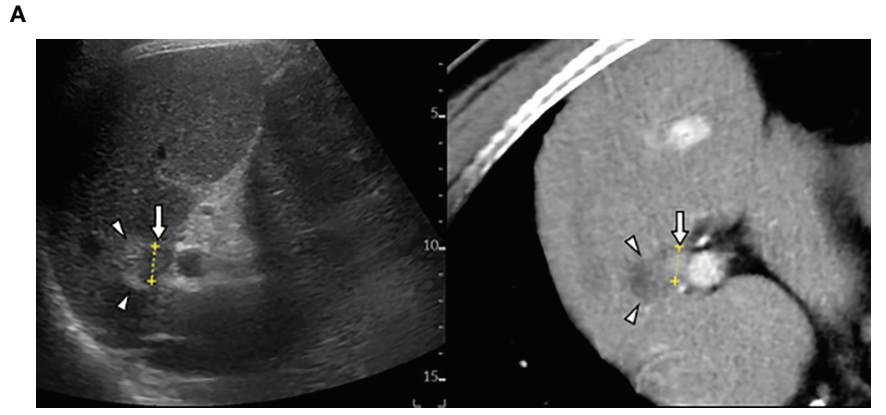

B

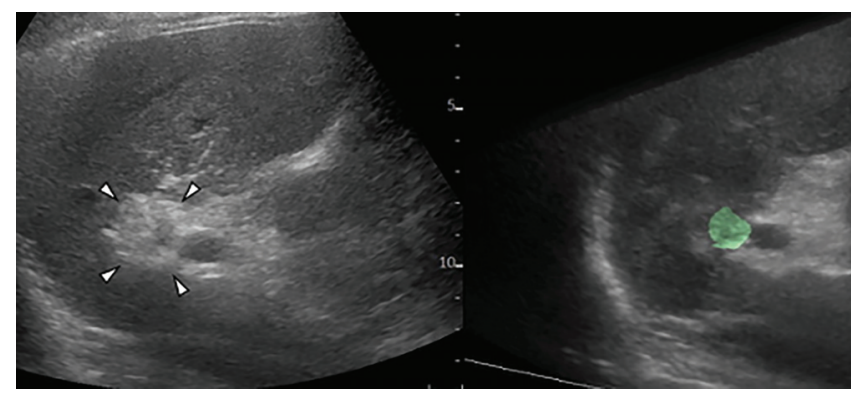

C

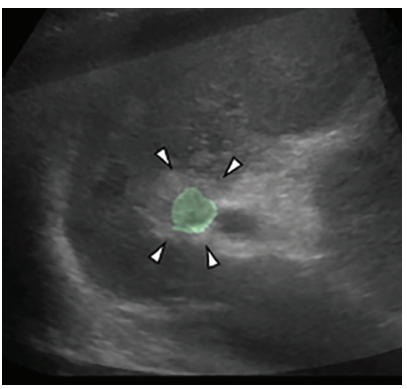

FIGURE 2 | US-US fusion imaging and US-US overlay fusion. (A) CT-US fused image shows locally progressed hepatocellular carcinoma (HCC) (arrows) touching ablative necrosis (arrow heads) due to previous ablation. (B) US-US fusion imaging displays HCC colorized as green before ablation (right) and ablative hyperechoic zone (arrowheads) due to the present ablaion (left). (C) US-US overlay fusion demonstrates the green colorized HCC inside the ablative hyperechoic zone concentrically.
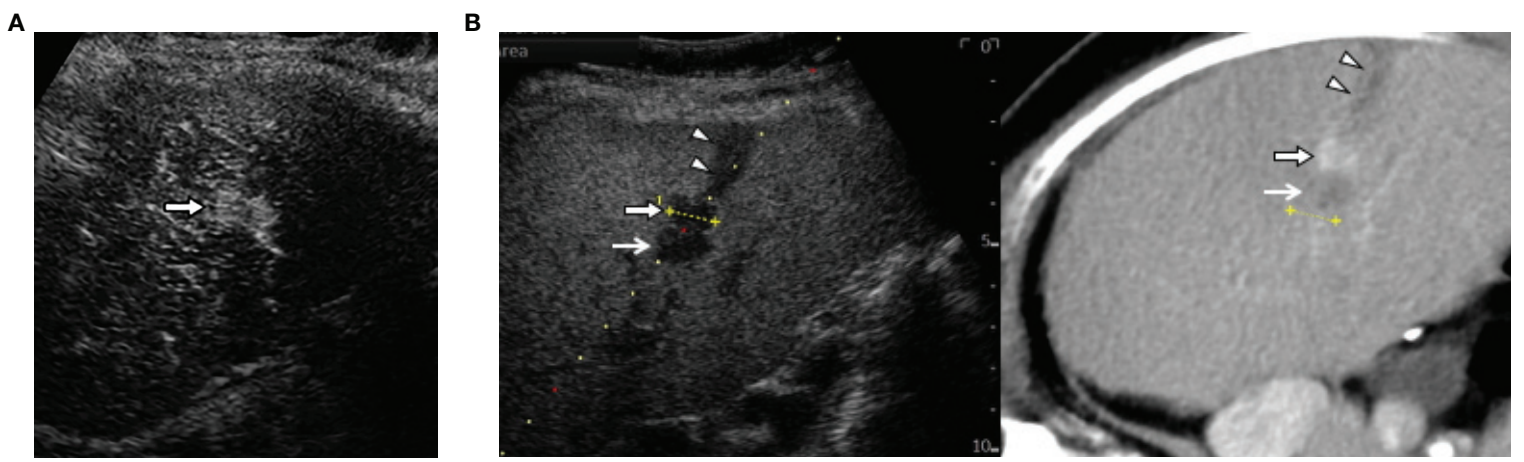

FIGURE 3 | The combination of contrast-enhanced US (CEUS) and fusion imaging. (A) CEUS with Sonazoid shows arterial enhancement of locally progressed hepatocellular carcinoma (HCC) (arrow). (B) The combination of Kupffer image on contrast-enhanced US (CEUS) and fused CT image demonstrates viable HCC (arrow) sandwiched between an ablated tract (arrow heads) and a necrotic tumor (open arrows). 


\section{THE COMBINATION GUIDANCE OF CEUS AND FUSION IMAGING}

Operators may attempt CEUS fused with CT/MR image when CT/ MR-US fusion imaging fails to identify HCCs. Either CEUS or fusion imaging provides an inadequately favorable condition for ablation therapy. Therefore, the combination of fusion imaging and CEUS is the last option (Figure 3). Even in difficult situations, we observed no significant differences in the number of treatment sessions required to obtain technical success of ablation between CEUS, fusion imaging, and the combination guidance (47). In addition, the combined guidance could be preferred for recurrent subcentimeter HCCs (48-50). This technique ablation may be expanded to intermediate stage HCC (51). However, CEUS has some detection limits for deep lesion, hypovascular HCC in a cirrhotic liver and lesions located in the subdiaphragrnatic regions. Therefore, we would like to recommend you choose fusion imaging guidance first.

\section{CONCLUSION}

CEUS and fusion imaging are relevant to identify HCC with poor conspicuity. Therefore, operators can perform ablation therapy with more confidence. CEUS/fusion imaging guidance has improved the clinical effectiveness of ablation therapy in poorly conspicuous HCC patients. However, CEUS or fusion imaging is limited in some situations. For example, HCC is unclear on CEUS either because the tumor location is deeper than $10 \mathrm{~cm}$, the CT/MRI and US images could not be finally wellmatched, or HCC is hidden behind bone or lung/bowl air. To overcome such situations, understanding the characteristics of each imaging guidance technique is key to identifying and managing poor conspicuous HCCs. No hostile relationship for ablation guidance between CEUS and fusion imaging. Occasionally, we can choose the combined guidance CEUS with fusion imaging in the most difficult situations. At least, we have to refrain from performing ablation therapy for poor conspicuous HCC with a simplistic strategy. CEUS/fusion imaging guided ablation therapy can provide longer recurrence-free survival rates and lower local tumor progression rates. Therefore, CEUS and fusion imaging can support the development of so-called "precise ablation."

\section{AUTHOR CONTRIBUTIONS}

All authors listed have made a substantial, direct, and intellectual contribution to the work and approved it for publication.

\section{REFERENCES}

1. Lin SM, Lin CJ, Lin CC, Hsu CW, Chen YC. Randomised controlled trial comparing percutaneous radiofrequency thermal ablation, percutaneous ethanol injection, and percutaneous acetic acid injection to treat hepatocellular carcinoma of $3 \mathrm{~cm}$ or less. Gut (2005) 54(8):1151-6. doi: 10.1136/gut.2004.045203

2. Chen MS, Li JQ, Zheng Y, Guo RP, Liang HH, Zhang YQ, et al. A prospective randomized trial comparing percutaneous local ablative therapy and partial hepatectomy for small hepatocellular carcinoma. Ann Surg (2006) 243:321-8. doi: 10.1097/01.sla.0000201480.65519.b8

3. Takayama T, Makuuchi M, Hasegawa K. Single HCC smaller than $2 \mathrm{~cm}$ : surgery or ablation?: surgeon's perspective. J Hepatobiliary Pancreat Sci (2010) 17(4):422-4. doi: 10.1007/s00534-009-0239-7

4. Peng ZW, Lin XJ, Zhang YJ, Liang HH, Guo RP, Shi M, et al. Radiofrequency ablation versus hepatic resection for the treatment of hepatocellular carcinomas $2 \mathrm{~cm}$ or smaller: a retrospective comparative study. Radiology (2012) 262(3):1022-33. doi: 10.1148/radiol.11110817

5. Feng K, Yan J, Li X, Xia F, Ma K, Wang S, et al. A randomized controlled trial of radiofrequency ablation and surgical resection in the treatment of small hepatocellular carcinoma. J Hepatol (2012) 57(4):794-802. doi: 10.1016/ j.jhep.2012.05.007

6. Kudo M. Management of Hepatocellular Carcinoma in Japan: Current Trends. Liver Cancer (2020) 9:1-5. doi: 10.1159/000505370

7. Lee MW, Lim HK, Kim YJ, Choi D, Kim YS, Lee WJ, et al. Percutaneous sonographically guided radio frequency ablation of hepatocellular carcinoma: causes of mistargeting and factors affecting the feasibility of a second ablation session. J Ultrasound Med (2011) 30(5):607-15. doi: 10.7863/jum.2011.30.5.607

8. Minami Y, Kudo M, Kawasaki T, Chung H, Ogawa C, Shiozaki H. Treatment of hepatocellular carcinoma with percutaneous radiofrequency ablation: usefulness of contrast harmonic sonography for lesions poorly defined with B-mode sonography. AJR Am J Roentgenol (2004) 183:153-6. doi: 10.2214/ ajr.183.1.1830153

9. Kim AY, Lee MW, Rhim H, Cha DI, Choi D, Kim YS, et al. Pretreatment evaluation with contrast-enhanced ultrasonography for percutaneous radiofrequency ablation of hepatocellular carcinomas with poor conspicuity on conventional ultrasonography. Korean J Radiol (2013) 14(5):754-63. doi: 10.3348/kjr.2013.14.5.754

10. Rajesh S, Mukund A, Arora A, Jain D, Sarin SK. Contrast-enhanced USguided radiofrequency ablation of hepatocellular carcinoma. J Vasc Interv Radiol (2013) 24(8):1235-40. doi: 10.1016/j.jvir.2013.04.013

11. Kudo M, Ueshima K, Osaki Y, Hirooka M, Aso K, Numata K, et al. for the SELECTED Study Group Japan. B-Mode ultrasonography versus contrastenhanced ultrasonography for surveillance of hepatocellular carcinoma: A prospective multicenter randomized controlled trial. Liver Cancer (2019) 8:271-80. doi: 10.1159/000501082

12. Liu F, Liu D, Wang K, Xie X, Su L, Kuang M, et al. Deep learning radiomics based on contrast-enhanced ultrasound might optimize curative treatments for very-early or early-stage hepatocellular carcinoma patients. Liver Cancer (2020) 9(4):397-413. doi: 10.1159/000505694

13. Shunichi S, Hiroko I, Fuminori M, Waki H. Definition of contrast enhancement phases of the liver using a perfluoro-based microbubble agent, perflubutane microbubbles. Ultrasound Med Biol (2009) 35:1819-27. doi: 10.1016/j.ultrasmedbio.2009.05.013

14. Dietrich CF, Nolsøe CP, Barr RG, Berzigotti A, Burns PN, Cantisani V, et al. Guidelines and Good Clinical Practice Recommendations for ContrastEnhanced Ultrasound (CEUS) in the Liver-Update 2020 WFUMB in Cooperation with EFSUMB, AFSUMB, AIUM, and FLAUS. Ultrasound Med Biol (2020) 46(10):2579-604. doi: 10.1016/j.ultrasmedbio.2020.04.030

15. Terminology and Diagnostic Criteria Committee. Japan Society of Ultrasonics in Medicine. Ultrasound diagnostic criteria for hepatic tumors. J Med Ultrason (2001) 41(1):113-23. doi: 10.1007/s10396-013-0500-1

16. Lee JY, Minami Y, Choi BI, Lee WJ, Chou YH, Jeong WK, et al. The AFSUMB Consensus Statements and Recommendations for the Clinical Practice of Contrast-Enhanced Ultrasound Using Sonazoid. Ultrasonography (2020) 39 (3):191-220. doi: 10.14366/usg.20057 
17. Kudo M. Breakthrough Imaging in Hepatocellular Carcinoma. Liver Cancer (2016) 5(1):47-54. doi: 10.1159/000367761

18. Minami Y, Kudo M. Contrast-enhanced ultrasonography with Sonazoid in hepatocellular carcinoma diagnosis. Hepatoma Res (2020) 6:46. doi: 10.20517/ 2394-5079.2020.32

19. Kudo M, Hatanaka K, Maekawa K. Newly developed novel ultrasound technique, defect reperfusion ultrasound imaging, using sonazoid in the management of hepatocellular carcinoma. Oncology (2010) 78:40-5. doi: $10.1159 / 000315229$

20. Kudo M. Defect reperfusion imaging with Sonazoid ${ }^{\circledR}$ : a breakthrough in hepatocellular carcinoma. Liver Cancer (2016) 5:1-7. doi: 10.1159/000367760

21. Kang TW, Lee MW, Song KD, Kim M, Kim SS, Kim SH, et al. Added Value of contrast-enhanced ultrasound on biopsies of focal hepatic lesions invisible on fusion imaging guidance. Korean J Radiol (2017) 18(1):152-61. doi: 10.3348/ kjr.2017.18.1.152

22. Korenaga K, Korenaga M, Furukawa M, Yamasaki T, Sakaida I. Usefulness of sonazoid contrast-enhanced ultrasonography for hepatocellular carcinoma: comparison with pathological diagnosis and superparamagnetic iron oxide magnetic resonance images. J Gastroenterol (2009) 44(7):733-41. doi: 10.1007/s00535-009-0053-7

23. Tanaka $\mathrm{H}$. Current role of ultrasound in the diagnosis of hepatocellular carcinoma. J Med Ultrason (2001) 47(2):239-55. doi: 10.1007/s10396-02001012-y

24. Wei Y, Ye Z, Yuan Y, Huang Z, Wei X, Zhang T, et al. A new diagnostic criterion with gadoxetic acid-enhanced MRI may improve the diagnostic performance for hepatocellular carcinoma. Liver Cancer (2020) 9(4):414-25. doi: $10.1159 / 000505696$

25. Minami Y, Kudo M, Chung H, Kawasaki T, Yagyu Y, Shimono T, et al. Contrast harmonic sonography-guided radiofrequency ablation therapy versus B-mode sonography in hepatocellular carcinoma: prospective randomized controlled trial. AJR Am J Roentgenol (2007) 188:489-94. doi: 10.2214/AJR.05.1286

26. Minami $Y$, Kudo $M$, Hatanaka $K$, Kitai $S$, Inoue $T$, Hagiwara $S$, et al. Radiofrequency ablation guided by contrast harmonic sonography using perfluorocarbon microbubbles (Sonazoid) for hepatic malignancies: an initial experience. Liver Int (2010) 30:759-64. doi: 10.1111/j.1478-3231. 2010.02226.x

27. Masuzaki R, Shiina S, Tateishi R, Yoshida H, Goto E, Sugioka Y, et al. Utility of contrast-enhanced ultrasonography with Sonazoid in radiofrequency ablation for hepatocellular carcinoma. J Gastroenterol Hepatol (2011) 26:759-64. doi: 10.1111/j.1440-1746.2010.06559.x

28. Dohmen T, Kataoka E, Yamada I, Miura K, Ohshima S, Shibuya T, et al. Efficacy of contrast-enhanced ultrasonography in radiofrequency ablation for hepatocellular carcinoma. Intern Med (2012) 51:1-7. doi: 10.2169/ internalmedicine.51.6042

29. Huang DY, Yusuf GT, Daneshi M, Ramnarine R, Deganello A, Sellars ME, et al. Contrast-enhanced ultrasound (CEUS) in abdominal intervention. Abdom Radiol (NY) (2018) 43:960-76. doi: 10.1007/s00261-018-1473-8

30. Minami $Y$, Kudo M. Ultrasound fusion imaging technologies for guidance in ablation therapy for liver cancer. J Med Ultrason (2001) 47(2):257-63. doi: 10.1007/s10396-020-01006-w

31. Hirooka M, Iuchi H, Kurose K, Kumagi T, Horiike N, Onji M. Abdominal virtual ultrasonographic images reconstructed by multi-detector row helical computed tomography. Eur J Radiol (2005) 53:312-7. doi: 10.1016/ j.ejrad.2004.03.026

32. Hirooka M, Iuchi H, Kumagi T, Shigematsu S, Hiraoka A, Uehara T, et al. Virtual sonographic radiofrequency ablation of hepatocellular carcinoma visualized on CT but not on conventional sonography. AJR Am J Roentgenol (2006) 186:S255-60. doi: 10.2214/AJR.04.1252

33. Minami $\mathrm{Y}$, Kudo $\mathrm{M}$, Chung $\mathrm{H}$, Inoue $\mathrm{T}$, Takahashi $\mathrm{S}$, Hatanaka $\mathrm{K}$, et al. Percutaneous radiofrequency ablation of sonographically unidentifiable liver tumors. Feasibility and usefulness of a novel guiding technique with an integrated system of computed tomography and sonographic images. Oncology (2007) 72 Suppl 1:111-6. doi: 10.1159/000111716

34. Kitada T, Murakami T, Kuzushita N, Minamitani K, Nakajo K, Osuga K, et al. Effectiveness of real-time virtual sonography-guided radiofrequency ablation treatment for patients with hepatocellular carcinomas. Hepatol Res (2008) 38 (6):565-71. doi: 10.1111/j.1872-034X.2007.00308.x
35. Minami $Y$, Chung $H$, Kudo M, Kitai S, Takahashi S, Inoue $T$, et al. Radiofrequency ablation of hepatocellular carcinoma: value of virtual CT sonography with magnetic navigation. AJR Am J Roentgenol (2008) 190(6): W335-341. doi: 10.2214/AJR.07.3092

36. Nakai M, Sato M, Sahara S, Takasaka I, Kawai N, Minamiguchi H, et al. Radiofrequency ablation assisted by real-time virtual sonography and CT for hepatocellular carcinoma undetectable by conventional sonography. Cardiovasc Intervent Radiol (2009) 32(1):62-9. doi: 10.1007/s00270-0089462-x

37. Song KD, Lee MW, Rhim H, Cha DI, Chong Y, Lim HK. Fusion imagingguided radiofrequency ablation for hepatocellular carcinomas not visible on conventional ultrasound. AJR Am J Roentgenol (2013) 201(5):1141-7. doi: 10.2214/AJR.13.10532

38. Ahn SJ, Lee JM, Lee DH, Lee SM, Yoon JH, Kim YJ, et al. Real-time US-CT/ MR fusion imaging for percutaneous radiofrequency ablation of hepatocellular carcinoma. J Hepatol (2017) 66(2):347-54. doi: 10.1016/ j.jhep.2016.09.003

39. Nishikawa H, Osaki Y, Iguchi E, Takeda H, Matsuda F, Nakajima J, et al. Radiofrequency ablation for hepatocellular carcinoma: the relationship between a new grading system for the ablative margin and clinical outcomes. J Gastroenterol (2013) 48(8):951-65. doi: 10.1007/s00535-0120690-0

40. Calandri M, Mauri G, Yevich S, Gazzera C, Basile D, Gatti M, et al. Fusion imaging and virtual navigation to guide percutaneous thermal ablation of hepatocellular carcinoma: a review of the literature. Cardiovasc Intervent Radiol (2019) 42(5):639-47. doi: 10.1007/s00270-019-02167-z

41. Toshikuni N, Tsutsumi M, Takuma Y, Arisawa T. Real-time image fusion for successful percutaneous radiofrequency ablation of hepatocellular carcinoma. J Ultrasound Med (2014) 33(11):2005-10. doi: 10.7863/ultra. 33.11.2005

42. Minami Y, Minami T, Chishina H, Kono M, Arizumi T, Takita M, et al. USUS Fusion Imaging in Radiofrequency Ablation for Liver Metastases. Dig Dis (2016) 34(6):687-91. doi: 10.1159/000448857

43. Minami $\mathrm{Y}$, Minami T, Hagiwara S, Ida H, Ueshima K, Nishida N, et al. Ultrasound-ultrasound image overlay fusion improves real-time control of radiofrequency ablation margin in the treatment of hepatocellular carcinoma. Eur Radiol (2018) 28(5):1986-93. doi: 10.1007/s00330-017$5162-8$

44. Xu EJ, Lv SM, Li K, Long YL, Zeng QJ, Su ZZ, et al. Immediate evaluation and guidance of liver cancer thermal ablation by three-dimensional ultrasound/ contrast-enhanced ultrasound fusion imaging. Int J Hyperthermia (2018) 34 (6):870-6. doi: 10.1080/02656736.2017.1373306

45. Lv S, Long Y, Su Z, Zheng R, Li K, Zhou H, et al. Investigating the accuracy of ultrasound-ultrasound fusion imaging for evaluating the ablation effect via special phantom-simulated liver tumors. Ultrasound Med Biol (2019) 45 (11):3067-74. doi: 10.1016/j.ultrasmedbio.2019.07.415

46. Minami $\mathrm{Y}$, Minami T, Takita M, Hagiwara S, Ida $\mathrm{H}$, Ueshima $\mathrm{K}$, et al Radiofrequency ablation for hepatocellular carcinoma: Clinical value of ultrasound-ultrasound overlay fusion for optimal ablation and local controllability. Hepatol Res (2020) 50(1):67-74. doi: 10.1111/hepr.13407

47. Minami T, Minami Y, Chishina H, Arizumi T, Takita M, Kitai S, et al. Combination guidance of contrast-enhanced US and fusion imaging in radiofrequency ablation for hepatocellular carcinoma with poor conspicuity on contrast-enhanced US/fusion imaging. Oncology (2014) 87 Suppl 1:55-62. doi: $10.1159 / 000368146$

48. Song KD, Lee MW, Rhim H, Kang TW, Cha DI, Sinn DH, et al. Percutaneous US/MRI fusion-guided radiofrequency ablation for recurrent subcentimeter hepatocellular carcinoma: technical feasibility and therapeutic outcomes. Radiology (2018) 288:878-86. doi: 10.1148/radiol.2018172743

49. Lee MW, Lim HK. Management of sub-centimeter recurrent hepatocellular carcinoma after curative treatment: Current status and future. World J Gastroenterol (2018) 24:5215-22. doi: 10.3748/wjg.v24.i46.5215

50. Numata K, Fukuda H, Morimoto M, Kondo M, Nozaki A, Oshima T, et al. Use of fusion imaging combining contrast-enhanced ultrasonography with a perflubutane-based contrast agent and contrast-enhanced computed tomography for the evaluation of percutaneous radiofrequency ablation of hypervascular hepatocellular carcinoma. Eur J Radiol (2012) 81(10):2746-53. doi: 10.1016/j.ejrad.2011.11.052 
51. Kariyama K, Nouso K, Wakuta A, Oonishi A, Toyoda H, Tada T, et al. Treatment of intermediate-stage hepatocellular carcinoma in Japan: Position of curative therapies. Liver Cancer (2020) 9:41-9. doi: 10.1159/000502479

Conflict of Interest: The authors declare that the research was conducted in the absence of any commercial or financial relationships that could be construed as a potential conflict of interest.
Copyright (C) 2021 Minami and Kudo. This is an open-access article distributed under the terms of the Creative Commons Attribution License (CC BY). The use, distribution or reproduction in other forums is permitted, provided the original author(s) and the copyright owner(s) are credited and that the original publication in this journal is cited, in accordance with accepted academic practice. No use, distribution or reproduction is permitted which does not comply with these terms. 